\title{
Follicular thyroid carcinoma
}

\author{
Manuel Sobrinho-Simões ${ }^{1,2,3}$, Catarina Eloy ${ }^{1,3,4}$, João Magalhães ${ }^{1,2,3}$, Cláudia Lobo ${ }^{5}$ \\ and Teresina Amaro ${ }^{5}$ \\ ${ }^{1}$ Institute of Molecular Pathology and Immunology of the University of Porto (IPATIMUP), Porto, Portugal; \\ ${ }^{2}$ Medical Faculty, Department of Pathology, University of Porto, Porto, Portugal; ${ }^{3}$ Department of Pathology, \\ Hospital S João; ${ }^{4}$ Department of Pathology, Dentistry Faculty, University of Porto, Porto, Portugal and \\ ${ }^{5}$ Department of Pathology, Portuguese Institute of Oncology Francisco Gentil, Porto, Portugal
}

\begin{abstract}
Follicular thyroid carcinoma is being diagnosed less and less frequently despite the increasing incidence of well-differentiated thyroid carcinomas everywhere. This review will discuss the reasons underlying such an observation focusing on the evolution of the morphological and immunohistochemical diagnostic criteria of follicular thyroid tumors. It will address the differential diagnosis between follicular carcinoma and three tumor types-follicular adenoma, follicular variant of papillary carcinoma and poorly differentiated carcinoma-as well as the problems raised by the newly described categories of follicular tumors: follicular tumor of uncertain malignant potential, well-differentiated tumor of uncertain malignant potential and welldifferentiated carcinoma, not otherwise specified. Finally, the prognostic and therapeutic significance of some promising molecular biomarkers will be discussed within the frame of the aforementioned histopathological classification.

Modern Pathology (2011) 24, S10-S18; doi:10.1038/modpathol.2010.133
\end{abstract}

Keywords: diagnosis; follicular thyroid carcinoma; molecular biomarkers; prognosis

In the respective chapter of the WHO Classification of Tumours of Endocrine Organs, follicular thyroid carcinoma (FTC) is defined as 'A malignant epithelial tumor showing follicular cell differentiation and lacking the diagnostic nuclear features of papillary thyroid carcinoma' (PTC). ${ }^{1}$ This definition is almost identical to the definition advanced by Rosai et $a l^{2}$ in the AFIP fascicle on Tumors of the Thyroid Gland: 'Follicular carcinoma is a malignant epithelial tumor showing evidence of follicular differentiation and not belonging to any of the other distinctive types of thyroid malignancy.'

Besides the point on follicular cell differentiation that does not usually raise any major difficulty, the two crucial issues of both definitions reside in the need to demonstrate malignancy and to exclude the presence of nuclear features typical of PTC. ${ }^{1-3}$

In this context, malignancy is equivalent to invasiveness (capsular and/or vascular penetration), which may be difficult to disclose with certainty. ${ }^{1-3}$ The situation is even worse regarding the tendency

Correspondence: Professor M Sobrinho-Simões, MD, PhD, Institute of Molecular Pathology and Immunology of the University of Porto (IPATIMUP), Rua Roberto Frias s/n, Porto 4200-465, Portugal.

E-mail: ssimoes@ipatimup.pt

Received 28 June 2010; accepted 28 June 2010 to overclassify the nuclear features of many follicular growth-patterned lesions as suggestive or indicative of those of the PTC nuclei; this trend is leading to an overdiagnosis of the follicular variant of PTC (FVPTC) and to an underdiagnosis of FTC. ${ }^{1,4}$

The utilization of more strict criteria for recognizing capsular and vascular invasion, and the aforementioned trend toward overdiagnosing FVPTC has led, together with the increasing number of PTC diagnoses (namely small or very small lesions), to a progressive reduction in the percentage of FTCs among well-differentiated thyroid carcinomas. ${ }^{1-4}$ For instance, the percentage of FTCs among welldifferentiated carcinomas decreased from $28.1 \%$ in the period $1983-1992$ to $6.5 \%$ in the period 1993-2007 in a very large series from Greece. ${ }^{5}$ In Japan, Kakudo et $a l^{6}$ recently reported that FTCs represented $9.7 \%$ among well-differentiated and moderately differentiated carcinomas. In a multiinstitutional study, we are performing in Porto, FTCs composed of 'normal' follicular cells or oncocytic cells represent $\sim 5 \%$ of clinically evident well-differentiated carcinomas (Magalhães et al, unpublished results). At present, in most US institutions, the frequency of FTC is even lower than that reported in the aforementioned series. 
Regardless of the differences from series to series, it is generally acknowledged that bona fide FTC is nowadays a relatively rare thyroid tumor in contrast to its high frequency in the series of the first half of last century. ${ }^{1-4}$

The prognosis of FTC depends on several factors: age of the patients, size and staging of the tumors, completeness of surgery and responsiveness to radioactive iodine. ${ }^{1-3,7}$ It also depends on the degree of invasiveness of tumors: minimally invasive carcinomas carry a much better prognosis than do widely invasive carcinomas. $^{1-3,7}$ The prognostic importance of angioinvasion has been recently acknowledged. ${ }^{1,7,8}$ The prognostic meaning of tissue and cell differentiation remains controversial, although it is generally accepted that FTCs composed of oncocytic cells and FTCs with a solid, trabecular or insular growth pattern respond less well to radioactive iodine and may carry a worse outcome. ${ }^{1-3,7,9,10}$ The coexistence of less well-differentiated areas in some FTCs raises the problem of the differential diagnosis between FTC and poorly differentiated thyroid carcinoma (PDTC). ${ }^{1,7,11}$

The above-mentioned diagnostic and prognostic issues will be addressed in this review using the chapter on follicular carcinoma of the WHO book, ${ }^{1}$ not only as a frame but also as a starting point for consolidating the classic criteria and discussing some recently advanced proposals. The molecular features will be integrated in the respective clinicopathological sections.

\section{Macroscopy and invasiveness}

Most FTCs are encapsulated solid tumors with a gray tan to brown color at the cut surface (Figure 1a). Minimally invasive FTCs are macroscopically indistinguishable from FA, except for capsular characteristics; the capsule tends to be thicker and more irregular in FTC than in FA. ${ }^{1-3,7}$ This is such an important feature that provided one is not dealing with a widely invasive FTC, the hypothesis of FTC should be ruled out if the tumor has no capsule or just a thin one. Widely invasive FTCs may occur as partially encapsulated tumors with extensive penetration of the capsule or as multinodular, bulking tumors without a capsule, occasionally showing permeation of the thyroid veins (Figure $1 \mathrm{~b}$ ). ${ }^{1-3,7}$

FTCs usually occur as single tumors. Multifocality and recurrence in the residual parenchyma after partial thyroidectomy are uncommon. The aneuploid pattern and the various molecular alterations detected in FA and FTC ${ }^{12-15}$ support the assumption that the development of such tumors is the end product of multiple oncogenic steps, thus justifying their usual appearance as single (unicentric) neoplasms. ${ }^{15}$ At variance with FTCs, PTCs occur frequently as multicentric neoplasms, which may be clonally independent from each other; ${ }^{15}$ taking this together with the fact that almost every PTC is
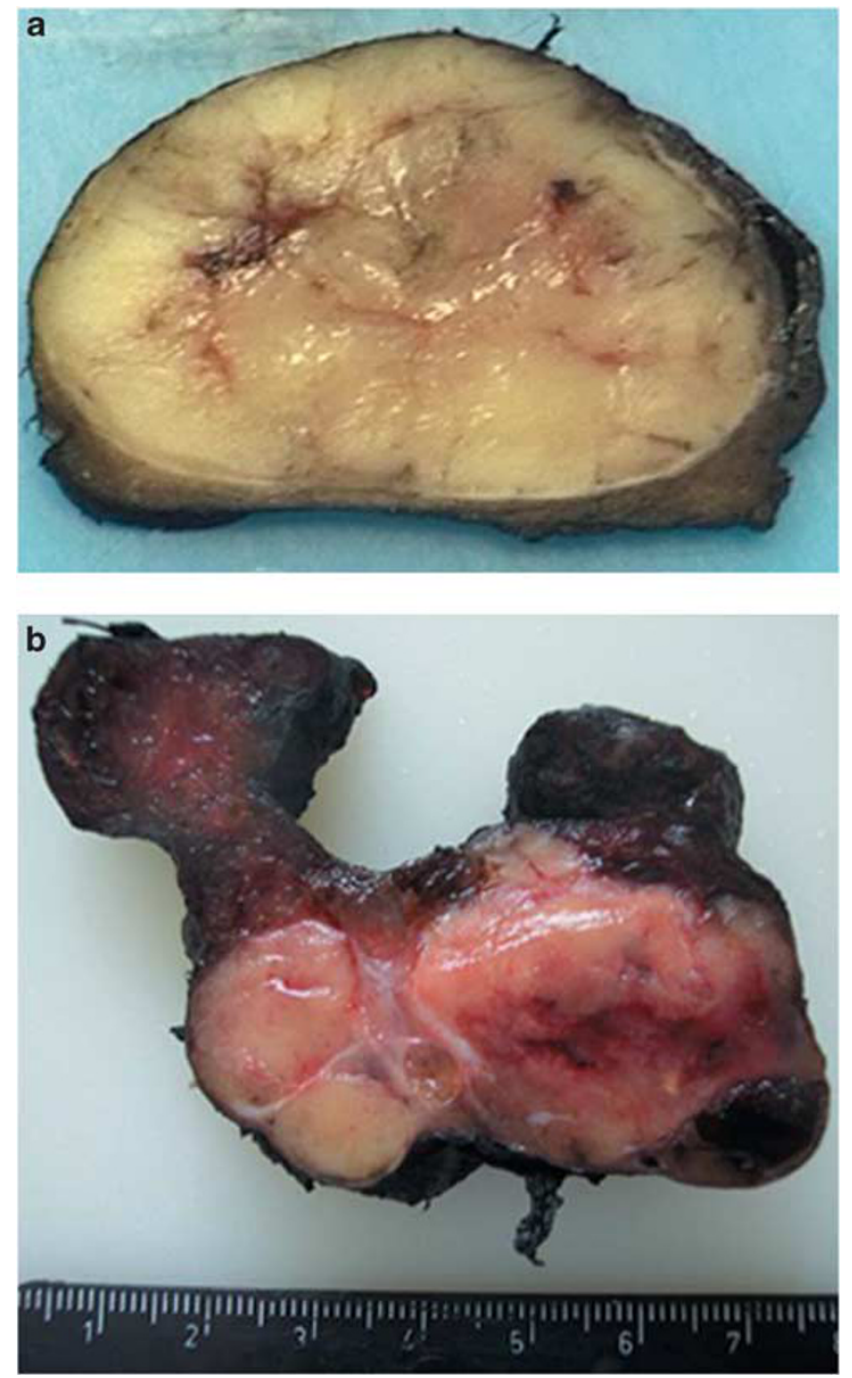

Figure 1 Macroscopic appearance of the two main types of follicular thyroid carcinoma. (a) Minimally invasive follicular thyroid carcinoma. (b) Widely invasive follicular thyroid carcinoma.

diploid or quasi-diploid and display microsatellite stability, it is tempting to advance that, in contrast to FTC, PTC tumorigenesis reflects the end product of a few oncogenic steps. ${ }^{15-17}$

The classification of FTCs in two categoriesminimally and widely invasive-has always created a sense of a 'pathological' gap: what about tumors that are 'simply' invasive? This gap has been filled with the creation of a third group of FTCs, the angioinvasive carcinomas. ${ }^{1,7}$ FTC is at present classified as 'minimally invasive without angioinvasion,' 'minimally invasive with angioinvasion' and 'widely invasive.' Most authors agree that for practical (prognostic and therapeutic) purposes, it is crucial to separate minimally invasive, not angioinvasive, FTCs which carry an excellent prognosis from angioinvasive FTCs regardless of whether it falls in the category of widely invasive FTCs. ${ }^{1-3,7,18-20}$ 
We believe that it is not worthwhile trying to count the number of vessels invaded by neoplastic cells in an attempt to establish a threshold separating two groups of prognostically different angioinvasive FTCs. Nikiforova et $a l^{21}$ have shown an association between the $P A X 8-P P A R \gamma$ rearrangement and very angioinvasive FTC ('overtly' invasive FTC), and a similar association was found by our group in FVPTC. $^{22}$ These findings reinforce the molecular similitude of FA/FTC and FVPTC (see below) and support the putative pathogenic importance of the $P A X 8-P P A R \gamma$ rearrangement in different types of follicular-patterned tumors, but there is not (yet?) enough evidence to use the detection of the $P A X 8$ $P P A R \gamma$ rearrangement for prognostic purposes. This is because first, $P A X 8-P P A R \gamma$ rearrangements are relatively frequent in follicular adenomas, ${ }^{23}$ and second, it is not known whether its presence carries prognostic information independently from vascular invasion.

In daily practice, we always try to detect at least one unequivocal focus of vascular invasion and, whenever this purpose is achieved, a diagnosis of angioinvasive FTC is made. ${ }^{24}$ Incidentally, whenever one identifies a focus of vascular invasion, several others are usually detected in the same section or in deeper sections.

The morphological criteria for identifying, for sure, signs of vascular invasion have been thoroughly described by LiVolsi, ${ }^{3}$ Rosai et $a l^{2}$ and Rosai, ${ }^{7}$ as well as in the WHO book. ${ }^{1}$ Although we agree with Mete and $\mathrm{Asa}^{8}$ about the ideal criteria for diagnosing vascular invasion (tumor cells invading a vessel wall associated with the thrombus adherent to intravascular tumor), we diagnose vascular invasion if we detect well-preserved neoplastic issue within a vein (Figure 2). Whenever facing the dilemma 'vascular invasion or not,' we do not rely on any immunostaining of endothelial cells; we search the additional sections of the capsule and stick to the following rule: If we do not detect any unequivocal signs of venous permeation, we diagnose the tumor as nonangionvasive (see below the point on 'follicular tumors of uncertain malignant potential').

\section{Histology and differential diagnosis}

Histologically, FTCs display variable morphology ranging from small/medium-sized follicles containing colloid to trabecular or solid growth pattern (Figure 2). The latter tends to be more frequent in widely invasive FTCs, but they can also occur in minimally invasive FTCs. It is exceedingly rare for a FTC to be composed of macrofollicles; in case one faces a capsular and/or vascular invasive follicular tumor composed of large follicles, with or without colloid, it is mandatory to look for the nuclear characteristics of neoplastic cells because the most likely diagnosis will be a (macro) FVPTC.
The cytological aspects can also vary a lot from case to case, and even within each case, without any clear-cut distinction between minimally and widely invasive FTCs. The same holds true regarding FA and adenomatous nodules of multinodular goiters which may present, either throughout the nodule or in some foci marked as cyto-arquitectural atypias. In other words, neither architectural nor cytological atypia are reliable criteria of malignancy. ${ }^{1-3,7,24}$ This and the need to identify unequivocal signs of invasion to make a diagnosis of FTC, renders fineneedle aspiration cytology and frozen sections useless or almost useless in these settings. ${ }^{1-3,7}$

\section{Differential Diagnosis between FA/Adenomatous Nodule and Minimally Invasive FTC}

Despite the huge amount of information on proliferation, apoptosis, cell-cycle differentiation and stromal markers obtained at the immunohistochemical level, ${ }^{1,15,25-31}$ the differential diagnosis between FA/adenomatous nodule and minimally invasive FTC still depends on the identification of histological signs of invasiveness. ${ }^{1-3,7,24}$ The search for such signs should be made in numerous H\&E-stained sections involving the capsule of the tumor. ${ }^{1-3,7,24}$ In our hands, as stressed above, immunostaining of capsular vessels does not provide useful diagnostic data. There is also no immunohistochemical or molecular feature that may be used as a reliable marker of invasion..$^{1,24,28}$

The most prominent molecular features of FTC are the prominence of aneuploidy and the high prevalence of $R A S$ mutations and of PAX8-PPAR $\gamma$ rearrangements, the latter as a result of a $2 ; 3$ translocation. ${ }^{12-15,32-34}$

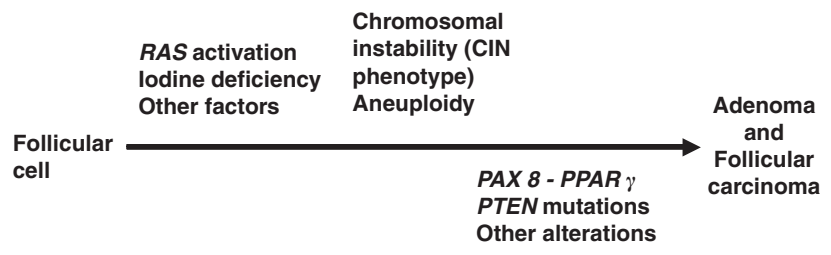

All the aforementioned molecular features (such as aneuploidy, RAS mutations, $P A X 8-P P A R \gamma$ rearrangements, etc.) are also frequently observed in FA, ${ }^{12-15,22,23,32-35}$ and are therefore almost useless for diagnostic purposes. In addition, the molecular approaches for diagnosing malignancy in follicular tumors using high-throughput technologies (DNA microarrays, massive sequencing, etc.) did not provide diagnostically sound results. ${ }^{36-38}$ The interesting preoperative diagnostic findings reported by Cerutti et $a l^{39}$ have not been confirmed in other series, and the same holds true for other studies. ${ }^{38}$

Taking into consideration the recently reported complex landscapes of somatic rearrangements in human breast cancer genomes ${ }^{40}$ and the more than 

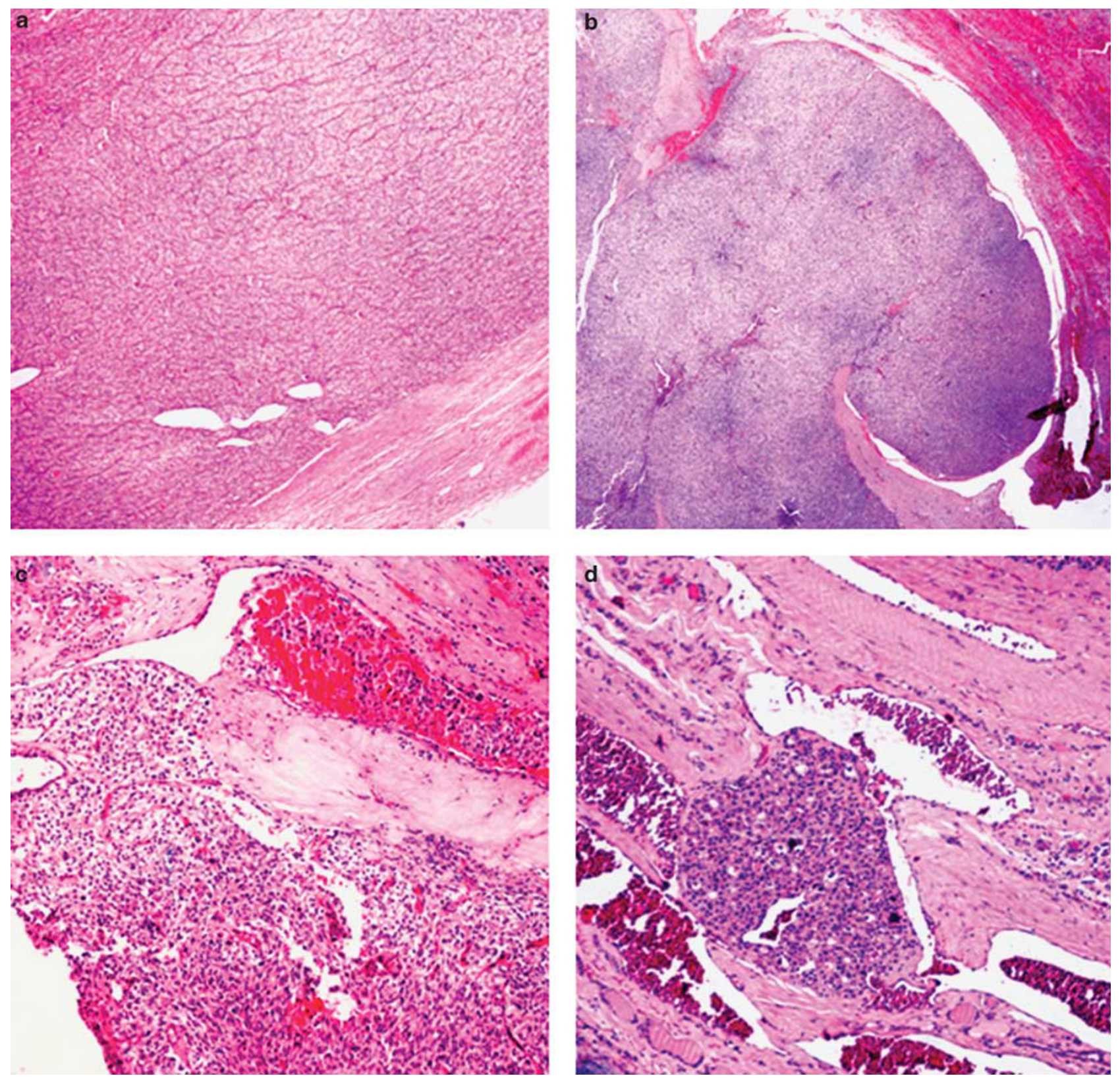

Figure 2 Histological appearance of a minimally invasive, angioinvasive, follicular thyroid carcinoma. (a) Microfollicular/solid pattern of growth of the neoplasia surrounded by a thick capsule (HE, $\times 4)$, (b) displaying, focally, capsular invasion (HE, $\times 4)$ and $(\mathbf{c}$ and $\mathbf{d})$ vascular invasion $(\mathrm{HE}, \times 100)$.

20000 or 30000 somatic mutations detected in individual human cancers, ${ }^{41,42}$ it may be anticipated that rather than searching for mutations by massive sequencing, it seems more sensible to progress in the diagnosis and prognosis of thyroid tumors by an integrated histological and molecular approach. ${ }^{28}$ The morphological frame is crucial for the topographical characterization of any tumor; this holds true particularly for follicular tumors of the thyroid as they epitomize the most important cancer properties: invasiveness in general and vascular invasiveness in particular. Besides 'topography,' the search for serum biomarkers may prove diagnostically useful in this setting. Despite our skepticism on the usefulness of high-throughput technologies in the differential diagnosis between FA and FTC, we anticipate that such methods will provide, in the future, meaningful information for predicting the risk of metastization and the sites of metastization.

For the time being, the histological analysis of exhaustive samples of the tumor capsule is thus necessary to make the differential diagnosis in borderline cases, namely by the identification of vascular invasiveness; if it is not possible to find unequivocal signs of invasiveness, then the designation of 'Follicular tumor of uncertain malignant 
potential' advanced by Williams ${ }^{43}$ is appropriate. ${ }^{1,7,24}$ We realize that the utilization of the term 'uncertain malignant potential' in a pathology report creates a big problem to clinicians. Such a term should be used as rarely as possible, not only because of its 'uncertainty' but also because it is not yet associated with any firmly established management protocol. Such diagnosis should only be made in cases in which one has a thorough evaluation of the whole capsule of the tumor, thus ruling out its utilization in most consultancy cases. The detection of several immunohistochemical and molecular markers has not proved to be diagnostically useful in this setting. ${ }^{26,44}$

In our institutions, follicular tumors of uncertain malignant potential are treated conservatively. Moreover, we think, like Rosai et al, ${ }^{2}$ Rosai, ${ }^{7}$ and other authors ${ }^{19,20}$ that follicular tumors in which capsular invasion is the sole sign of malignancy should also be treated conservatively (see below), but these issues need to be addressed in Multidisciplinary Consensus Conferences to produce management protocols.

\section{Differential Diagnosis between Minimally Invasive FTC and Encapsulated FVPTC}

At present, this is the most frequent reason for consultancy in thyroid pathology. A few years ago, it used to be the identification of signs of capsular and/or vascular invasion, whereas nowadays it is 'Whether or not the nuclear characteristics of the neoplastic cells support the diagnosis of PTC. ${ }^{1-3,7,24,45}$

The diagnosis of encapsulated FVPTC has probably become the hottest topic in thyroid pathology mainly because of the too-liberal utilization of the PTC nuclear criteria. It should be stressed that this dilemma is restricted to the encapsulated type of FVPTC as the other two types of FVPTCs (such as poorly circumscribed and multinodular/diffuse types) do not usually raise diagnostic problems. ${ }^{21,24,45}$

We have been stressing, since 2002, the existence of the aforementioned three types of FVPTCs. ${ }^{46}$ These types apparently have different natural histories and display quite different patterns of metastization. ${ }^{15,22,24,34,46,47}$ Poorly circumscribed/infiltrative FVPTCs metastasize, like conventional PTCs, to regional lymph nodes; ${ }^{1-3,44,46}$ encapsulated FVPTCs tend to give rise, whenever displaying angioinvasiveness, to lung and bone metastases without concurrent nodal metastases; ${ }^{47,48}$ and multinodular/diffuse FVPTCs are characterized by the frequent metastatic involvement of bones and lungs together with lymph nodes. ${ }^{49}$ The peculiar bloodborn metastatic pattern of some cases of FVPTCs had been highlighted many years ago by Rosai and colleagues $^{50}$ and Rosai. ${ }^{51}$

Besides the low frequency and type of the BRAF mutation, ${ }^{34,52,53}$ there are other molecular features of FVPTCs in general, and of encapsulated FVPTCs in particular, that differ from those of conventional PTCs. Some of those features resemble the molecular alterations that are characteristic of FA and FTC. ${ }^{1}$ E-cadherin immunoexpression in encapsulated FVPTCs resembles that of follicular tumors and normal thyroid rather than that of conventional PTCs. ${ }^{54,55}$ The frequency of RAS mutations is much higher in FVPTCs than in conventional PTCs, ${ }^{22,56,57}$ thus also contributing to approach the FVPTC to FA and FTC. ${ }^{2,51}$ Similar prevalences of RAS mutations were detected in FA (33\%), FTC $(22 \%)$ and FVPTC $(25 \%){ }^{22}$ Roque et $a l^{58}$ described, in 2001, using conventional cytogenetics, the $\mathrm{t}(2 ; 3)(\mathrm{q} 12 ; \mathrm{p} 25)$ in one case of FVPTC, and Wreesmann et al ${ }^{59}$ using immunohistochemistry demonstrated, in 2004, overexpression of PPAR $\gamma$ in three cases of FVPTCs. Castro et $\mathrm{al}^{22}$ detected the presence of PAX8-PPAR $\gamma$ rearrangement in $37 \%$ of the cases of encapsulated FVPTCs, $33 \%$ of FAs and $45 \%$ of FTCs, in contrast to its absence in conventional PTCs. ${ }^{54,55}$ The results obtained by Castro et $a^{22}$ in FVPTCs probably reflect the stringent criteria used in the selection of cases of FVPTCs, excluding all tumors with a follicular/ papillary growth pattern.

Taken together, the aforementioned data support the conclusion that FVPTCs show many genetic differences from conventional PTCs ${ }^{22,59,60}$ and suggest that at least a subset of FVPTC that apparently corresponds to the encapsulated type of FVPTC shares some of the molecular features of FTC. ${ }^{22,57}$ The same holds true in FTC and FVPTC arising in struma ovarii cases. ${ }^{61}$

It remains to be seen whether the subset of FVPTC displaying genetic alterations of the FTC type encompasses cases particularly prone to give rise to lung and/or bone metastases. It also remains unclarified whether cases of encapsulated FVPTC lacking signs of vascular invasion metastasize to the lungs or bones regardless of whether having the $P A X 8-P P A R \gamma$ rearrangement or other genetic alterations. The results obtained so far in our retrospective study of 239 cases of well-differentiated thyroid carcinomas diagnosed in the Hospital S. João and Porto Cancer Institute, from 1978 to 2003, which displayed nodal and/or blood born metastases, has shown that in this group there was not a single case of follicular tumor of uncertain malignant potential or a well-differentiated tumor of uncertain malignant potential, nor any case of minimally invasive, non angioinvasive FTC or encapsulated, noninvasive FVPTC (Magalhães et al, unpublished results).

The problem of disclosing the malignant potential of encapsulated, nonangioinvasive FVPTC and, based on such evaluation, to decide the best therapy for such cases is similar to the discussion on the clinical malignancy and therapy selection for minimally invasive FTC with capsular invasion alone (see above). The evidence on record suggests that for both situations, lobectomy or lobectomy plus isthmectomy will be enough. ${ }^{18,20,62,63}$ 
Summing up, there are two separate problems in the diagnosis of encapsulated tumors with follicular architecture: to decide whether minor nuclear changes of the PTC type justify a diagnosis of FVPTC and to decide whether a minor degree of capsular penetration justifies a diagnosis of malignancy. ${ }^{43}$ For the moment and although there is no additional evidence from the molecular pathology side, we agree with Williams and the Chernobyl Study Group $^{43}$ that it is more appropriate to recognize the difficulty in deciding whether the nuclei are typical than to arbitrarily place welldifferentiated encapsulated tumors with a follicular architecture, in which minor nuclear changes are the only indicator of a PTC, in a definite malignant or a definite benign category. ${ }^{43}$ Like Williams ${ }^{43}$ and many other authors in Europe, ${ }^{1,7,24,26,44,46}$ we refer to these tumors simply as 'well differentiated' without specifying either follicular or papillary, and use the terms 'well-differentiated tumor of uncertain malignant potential' when there is no definite evidence of invasion, and 'well-differentiated carcinoma, NOS' when invasion is present. ${ }^{1,24,64}$ The term 'welldifferentiated tumor of uncertain malignant potential' should be used as rarely as possible and should never be considered as a substitute for adequate sampling of any follicular-patterned thyroid tumor. ${ }^{1,65}$ In our experience, and in that of other groups, ${ }^{19,20,62,63}$ these tumors are adequately treated with a conservative approach (lobectomy or lobectomy plus isthmectomy), but one still needs firmly established management protocols. Although we do not sympathize with the designation 'well-differentiated carcinoma, NOS,' we use it occasionally for two main reasons: some follicular-patterned carcinomas of the thyroid are difficult to characterize because they have intermediate nuclei or different types of nuclei, and the treatment is similar for 'conventional PTC,' 'angioinvasive FTC' and 'angioinvasive, well-differentiated carcinoma, NOS.'

\section{Differential Diagnosis between Widely Invasive FTC and Poorly Differentiated Thyroid Carcinoma}

In the WHO book, ${ }^{64}$ PDTC has been recognized as a histopathological entity. The absence of clear diagnostic criteria for PDTC has been recently solved by Volante et $a{ }^{11}$ who described such criteria and advanced an algorithmic diagnostic approach for these tumors.

Despite the aforementioned developments in the diagnosis of PDTCs, it may be difficult to separate them from FTCs with a predominant trabecular/ insular/solid growth pattern. ${ }^{11,60,61}$ The same holds true regarding the separation of PDTCs from PTCs predominantly composed of trabecular, insular or solid areas whenever the nuclei of neoplastic cells superficially resemble the PTC nuclei. ${ }^{11,64,65}$

In both instances (DD with PTC and FTC), the foci of necrosis and high mitotic rate may be used in individual cases as signs suggestive of PDTC but should never be considered as absolute criteria. ${ }^{11,64,65}$ The evaluation of the nuclear features is crucial whenever one faces the differential diagnosis with PTC; if the nuclei in the 'poorly differentiated' areas are of the PTC type, then we stick to the diagnosis of PTCs with trabecular or solid areas because the prognosis is much better than that of PDTCs. ${ }^{11,64}$

From a pragmatic standpoint, the separation of PDCs from FTCs only matters when one is dealing with a minimally invasive neoplasm, as widely invasive FTCs carry a prognosis that is similar to that of PDTCs and should be treated just as radically as them. ${ }^{11,64,65}$

\section{Oncocytic (Hürthle Cell) Variant of FTC}

Tumors with oncocytic (Hürthe cell) features may be benign (oncocytic variant of FA) or malignant (oncocytic variant of PTC and oncocytic variant of FTC). Medullary and poorly differentiated carcinomas may also display oncocytic features. ${ }^{9,10,66,67}$

Hürthle cells are stuffed with the mitochondria with morphological, functional and genetic abnormalities. $^{9,10,66,67}$ The accumulation of the mitochondria may be due to a primary alteration of mitochondrial DNA or of nuclear DNA that encode mitochondrial enzymes. ${ }^{9,10,68,69}$ Regardless of the mechanism(s) involved in the process, a deficient mitochondrial function leads to an increased number of mitochondria through the stimulation of mitochondrial proliferation..$^{9,10,68,69}$

It has been shown that most of the typical molecular features of conventional PTCs and FTCs are also present in their oncocytic counterpart. ${ }^{15,35,52,68,69}$ For practical purposes, the oncocytic appearance is believed to represent a phenotype that is superimposed to the genotypic and histopathological features of thyroid tumors.

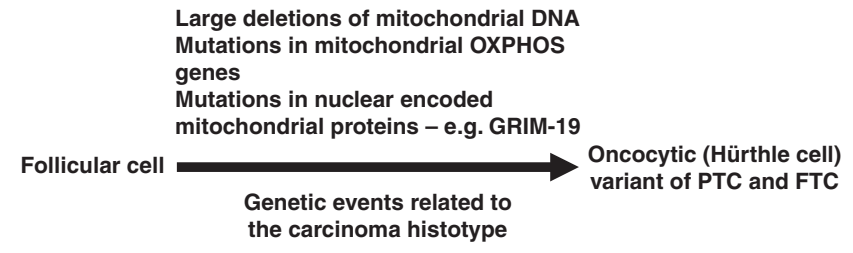

The criteria used in the diagnosis of the oncocytic variants of PTCs and FTCs are those used in the diagnosis of conventional tumors, ie, mainly the nuclear characteristics in PTCs and the signs of capsular and/or vascular invasion in FTCs. The prognosis of patients with the oncocytic variant of PTCs or FTCs is similar to that of patients with the respective conventional carcinomas provided the age of the patients and the staging of the tumors are comparable. ${ }^{1,69-71}$ From a clinical standpoint, the negative aspect of oncocytic carcinomas of the thyroid is their lesser ability to trap iodine, 
thus rendering them less responsive to radioactive iodine. ${ }^{1,10,72,73}$

Although it is now widely accepted that many oncocytic tumors of the thyroid are benign (nodular goiters and FA with oncocytic features), one should keep in mind that the percentage of oncocytic tumors displaying signs of invasiveness is higher than that occurring in tumors without oncocytic features. That is the reason why one should search actively for capsular and vascular invasion, and for the PTC nuclei, whenever dealing with any oncocytic tumor that looks benign at a first glance. ${ }^{1-3,7,65}$

\section{Acknowledgements}

This review is partly based on a clinico-pathological study supported by Fundação Calouste Gulbenkian (Project: 'Identificação de factores de prognóstico e de selecção terapêutica em carcinomas diferenciados da Tireoide'). The support of the Consortium IPATIMUP-IPO-Porto is also gratefully acknowledged.

\section{Disclosure/conflict of interest}

The authors declare no conflict of interest.

\section{References}

1 Sobrinho Simões M, Asa SL, Kroll TG, et al. Follicular carcinoma. In: DeLellis RA, Lloyd RV, Heitz PU and Eng C (eds). WHO Classification of Tumors. Pathology and Genetics. Tumours of Endocrine Organs. IARC Press: Lyon, France, 2004. pp 67-76.

2 Rosai J, Carcangiu ML, DeLellis R. Tumors of the Thyroid Gland: Atlas of Tumor Pathology. Vol 5. 3rd series Armed Forces Institute of Pathology: Washington, DC, 1992. pp 343.

3 LiVolsi VA. Surgical Pathology of The Thyroid. WB Saunders: Philadelphia, PA, 1990.

4 LiVolsi VA, Asa SL. The demise of follicular carcinoma of the thyroid gland. Thyroid 1994;4:233-236.

5 Alevizaki M, Papageorgiou G, Rentziou G, et al. Increasing prevalence of papillary thyroid carcinoma in recent years in Greece: the majority are incidental. Thyroid 2009;19:749-754.

6 Kakudo K, Bai Y, Katayama S, et al. Classification of follicular cell tumors of the thyroid gland: analysis involving Japanese patients from one institute. Pathol Int 2009;59:359-367.

7 Rosai J, editor Rosai and Ackerman's Surgical Pathology. Vol 1. 9th edn. Mosby: London, 2004, pp 1482.

8 Mete O, Asa SL. Definition and clinical significance of vascular invasion in thyroid carcinomas derived from follicular epithelium. Mod Pathol 2010;23(Supp 1):131A.

9 Maximo V, Sobrinho-Simoes M. Hurthle cell tumours of the thyroid. A review with emphasis on mitochondrial abnormalities with clinical relevance. Virchows Arch 2000;437:107-115.

10 Máximo V, Soares P, Sobrinho Simoes M. Mitochondria and cancer. Virchows Arch 2009;454:481-495.
11 Volante M, Collini P, Nikiforov YE, et al. Poorly differentiated thyroid carcinoma: the Turin proposal for the use of uniform diagnostic criteria and an algorithmic diagnostic approach. Am J Surg Pathol 2007;31:1256-1264.

12 Johannessen JV, Sobrinho-Simões M, Lindmo T, et al. The diagnostic value of flow cytometric DNA measurements in selected disorders of the human thyroid. Am J Clin Pathol 1982;77:20-25.

13 Castro P, Sansonetty F, Soares P, et al. Fetal adenomas and minimally invasive follicular carcinomas of the thyroid frequently display a triploid or near triploid DNA pattern. Virchows Arch 2001;438:336-342.

14 Castro P, Eknaes M, Teixeira MR, et al. Adenomas and follicular carcinomas of the thyroid display two major patterns of chromosomal changes. J Pathol 2005;206: 305-311.

15 Sobrinho-Simões M, Preto A, Rocha AS, et al. Molecular pathology of well-differentiated thyroid carcinomas. Virchows Arch 2005;447:787-793.

16 Johannessen JV, Sobrinho-Simões M, Tangen KO, et al. A flow cytometric deoxyribonucleic acid analysis of papillary thyroid carcinoma. Lab Invest 1981;45: 336-341.

17 Soares P, dos Santos NR, Seruca R, et al. Benign and malignant thyroid lesions show instability at microsatellite loci. Eur J Cancer 1997;33:293-296.

18 Lang W, Choritz H, Hundeshagen H. Risk factors in follicular thyroid carcinomas. A retrospective followup study covering a 14-year period with emphasis on morphological findings. Am J Surg Pathol 1986;10: 246-255.

19 van Heerden JA, Hay ID, Goellner JR, et al. Follicular thyroid carcinoma with capsular invasion alone: a nonthreatening malignancy. Surgery 1992;112: 1130-1136.

20 Thompson LD, Wieneke JA, Paal E, et al. A clinicopathologic study of minimally invasive follicular carcinoma of the thyroid gland with a review of the English literature. Cancer 2001;91:505-524.

21 Nikiforova MN, Lynch RA, Biddinger PW, et al. RAS point mutations and PAX8-PPAR gamma rearrangement in thyroid tumors: evidence for distinct molecular pathways in thyroid follicular carcinoma. J Clin Endocrinol Metab 2003;88:2318-2326.

22 Castro P, Rebocho AP, Soares RJ, et al. PAX8-PPAR $\gamma$ rearrangement is frequently detected in the follicular variant of papillary thyroid carcinoma. J Clin Endocrinol Metab 2006;91:213-220.

23 Marques AR, Espadinha C, Catarino AL, et al. Expression of PAX8-PPAR gamma 1 rearrangements in both follicular thyroid carcinomas and adenomas. J Clin Endocrinol Metab 2002;87:3947-3952.

24 Fonseca E, Soares $\mathrm{P}$, Cardoso-Oliveira $\mathrm{M}$, et al. Diagnostic criteria in well-differentiated thyroid carcinomas. Endocr Pathol 2006;17:109-117.

25 de Matos PS, Ferreira AP, de Oliveira FF, et al. Usefulness of HBME-1, cytokeratin 19 and galectin-3 immunostaining in the diagnosis of thyroid malignancy. Histopathology 2005;47:391-401.

26 Prasad ML, Pellegata NS, Huang Y, et al. Galectin-3, fibronectin-1, CITED-1, HBME1 and cytokeratin-19 immunohistochemistry is useful for the differential diagnosis of thyroid tumors. Mod Pathol 2005;18: 48-57.

27 Papotti M, Rodriguez J, De Pompa R, et al. Galectin-3 and HBME-1 expression in well-differentiated thyroid 
tumors with follicular architecture of uncertain malignant potential. Mod Pathol 2005;18:541-546.

28 Cho Mar K, Eimoto T, Tateyama H, et al. Expression of matrix metalloproteinases in benign and malignant follicular thyroid lesions. Histopathology 2006;48: 286-294.

29 Cho Mar K, Eimoto T, Nagaya S, et al. Cell proliferation marker MCM2, but not Ki67, is helpful for distinguishing between minimally invasive follicular carcinoma and follicular adenoma of the thyroid. Histopathology 2006;48:801-807.

30 Rigaud C, Bogomoletz WV. Apparent lack of usefulness of monoclonal antibody Ki-67 in thyroid tumour pathology. Relation to histological typing and classification. Pathol Res Pract 1991;187:198-200.

31 Soares P, Sobrinho-Simöes M. Proliferative activity of human thyroid tumors evaluated by proliferating cell nuclear antigen/cyclin immunohistochemical studies. Cancer 1994;73:2879-2881.

32 Namba H, Rubin SA, Fagin JA. Point mutations of ras oncogenes are an early event in thyroid tumorigenesis. Mol Endocrinol 1990;4:1474-1479.

33 Kroll TG, Sarraf P, Pecciarini L, et al. PAX8-PPARgamma1 fusion oncogene in human thyroid carcinoma [corrected]. Science 2000;289:1357-1360.

34 Sobrinho-Simões M, Máximo V, Rocha AS, et al. Intragenic mutations in thyroid cancer. Endocrinol Metab Clin North Am 2008;37:333-362.

35 Cheung CC, Ezzat S, Ramyar L, et al. Molecular basis of Hurthle cell papillary thyroid carcinoma. J Clin Endocrinol Metab 2000;85:878-882.

36 Mazzanti C, Zeiger MA, Costouros NG, et al. Using gene expression profiling to differentiate benign versus malignant thyroid tumors. Cancer Res 2004;64: 2898-2903.

37 Giordano TJ. Genome-wide studies in thyroid neoplasia. Endocrinol Metab Clin North Am 2008;37:311-331.

38 Eszlinger M, Krohn K, Hauptmann S, et al. Perspectives for improved and more accurate classification of thyroid epithelial tumors. J Clin Endocrinol Metab 2008;93:3286-3294.

39 Cerutti JM, Delcelo R, Amadei MJ, et al. A preoperative diagnostic test that distinguishes benign from malignant thyroid carcinoma based on gene expression. J Clin Invest 2004;113:1234-1242.

40 Stephens PJ, McBride DJ, Lin ML, et al. Complex landscapes of somatic rearrangement in human breast cancer genomes. Nature 2009;462:1005-1010.

41 Pleasance ED, Stephens PJ, O'Meara S, et al. A smallcell lung cancer genome with complex signatures of tobacco exposure. Nature 2010;463:184-190.

42 Pleasance ED, Cheetham RK, Stephens PJ, et al. A comprehensive catalogue of somatic mutations from a human cancer genome. Nature 2010;463:191-196.

43 Williams ED. Guest editorial: two proposals regarding the terminology of thyroid tumors. Int J Surg Pathol 2003;8:181-183.

44 Hofman V, Lassalle S, Bonnetaud C, et al. Thyroid tumours of uncertain malignant potential: frequency and diagnostic reproducibility. Virchows Arch 2009; 455:21-33.

45 Hapke MR, Dehner LP. The optically clear nucleus. A reliable sign of papillary carcinoma of the thyroid? Am J Surg Pathol 1979;3:31-38.

46 Castro P, Fonseca E, Magalhães J, et al. Follicular, papillary, and 'hybrid' carcinomas of the thyroid. Endocr Pathol 2002;13:313-320.
47 Liu J, Singh B, Tallini G, et al. Follicular variant of papillary thyroid carcinoma: a clinicopathologic study of a problematic entity. Cancer 2006;107:1255-1264.

48 Baloch ZW, LiVolsi VA. Encapsulated follicular variant of papillary thyroid carcinoma with bone metastases. Mod Pathol 2000;13:861-865.

49 Ivanova R, Soares P, Castro P, et al. Diffuse (or multinodular) follicular variant of papillary thyroid carcinoma: a clinicopathologic and immunohistochemical analysis of ten cases of an aggressive form of differentiated thyroid carcinoma. Virchows Arch 2002;440:418-424.

50 Carcangiu M, Zampi G, Pupi A, et al. Papillary carcinoma of the thyroid. A clinicopathologic study of 241 cases treated at the University of Florence, Italy. Cancer 1985;55:805-828.

51 Rosai J, Zampi G, Carcangiu ML. Papillary carcinoma of the thyroid. A discussion of its several morphologic expressions, with particular emphasis on the follicular variant. Am J Surg Pathol 1983;7:809-817.

52 Trovisco V, Vieira de Castro I, Soares P, et al. BRAF mutations are associated with some histological types of papillary thyroid carcinoma. J Pathol 2004;202: 247-251.

53 Rocha AS, Soares P, Seruca R, et al. Abnormalities of E-cadherin/catenin complex in classical papillary thyroid carcinoma and its diffuse sclerosing variant. J Pathol 2001;194:358-366.

54 Soares P, Berx G, van Roy F, et al. E-cadherin gene alterations are rare events in thyroid tumors. Int J Cancer 1997;70:32-38.

55 Castro P, Soares P, Gusmão L, et al. H-RAS 81 polymorphism is significantly associated with aneuploidy in follicular tumors of the thyroid. Oncogene 2006;25:4620-4627.

56 Zhu Z, Gandhi M, Nikiforova MN, et al. Molecular profile and clinical-pathologic features of the follicular variant of papillary thyroid carcinoma. An unusually high prevalence of ras mutations. Am J Clin Pathol 2003;120:71-77.

57 Rivera M, Ricarte-Filho J, Knauf J, et al. Molecular genotyping of papillary thyroid carcinoma follicular variant according to its histologic subtypes (encapsulated vs non-encapsulated infiltrative) reveals distinct BRAF and RAS mutations patterns. Mod Pathol 2010;23(Supp 1):pp132A.

58 Roque L, Nunes VM, Ribeiro C, et al. Karyotypic characterization of papillary thyroid carcinomas. Cancer 2001;92:2529-2538.

59 Wreesmann VB, Ghossein RA, Hezel M, et al. Follicular variant of papillary thyroid carcinoma: genome-wide appraisal of a controversial entity. Genes Chromosomes Cancer 2004;40:355-364.

60 Finn SP, Smyth P, Cahill S, et al. Expression microarray analysis of papillary thyroid carcinoma and benign thyroid tissue: emphasis on the follicular variant and potential markers of malignancy. Virchows Arch 2007;450:249-260.

61 Celestino R, Magalhães J, Castro P, et al. A follicular variant of papillary thyroid carcinoma in struma ovarii. Case report with unique molecular alterations. Histopathology 2009;55:482-487.

62 Widder S, Guggisberg K, Khalil M, et al. A pathologic re-review of follicular thyroid neoplasms: the impact of changing the threshold for the diagnosis of the follicular variant of papillary thyroid carcinoma. Surgery 2008;144:80-85. 
63 Piana S, Frasoldati A, Di Felice E, et al. Encapsulated well-differentiated follicular-patterned thyroid carcinomas do not play a significant role in the family rates from thyroid carcinoma. Am J Surg Pathol 2010;34:868-872.

64 Sobrinho-Simoes M, Albores-Saavedra J, Tallini G, et al. Poorly differentiated carcinoma. In: DeLellis RA, Lloyd RV, Heitz PU, Eng C, (eds). WHO Classification of Tumors. Pathology and Genetics. Tumours of Endocrine Organs. IARC Press: Lyon, France, 2004. pp 73-76.

65 Sobrinho-Simões M, Magalhães J, Fonseca E, et al. Diagnostic pitfalls of thyroid pathology. Curr Diagnost Pathol 2005;11:52-59.

66 Sobrinho-Simoes M, Maximo V, Vieira de Castro I, et al. Hurthle (oncocytic) cell tumors of thyroid: etiopathogenesis, diagnosis and clinical significance. Int J Surg Pathol 2005;13:29-35.

67 Sobrinho-Simoes MA, Nesland JM, Holm R, et al. Hurthle cell and mitochondrion-rich papillary carcinomas of the thyroid gland: an ultrastructural and immunocytochemical study. Ultrastruct Pathol 1985;8:131-142.

68 Máximo V, Botelho T, Capela J, et al. Somatic and germline mutation in GRIM-19, a dual function gene involved in mitochondrial metabolism and cell death, is linked to mitochondrion-rich (Hürthle cell) tumours of the thyroid. Br J Cancer 2005;92:1892-1898.

69 Máximo V, Soares P, Lima J, et al. Mitochondrial DNA somatic mutations (point mutations and large deletions) and mitochondrial DNA variants in human thyroid pathology. A study with emphasis on Hürthle cell tumors. Am J Pathol 2002;160:1857-1865.

70 Trovisco V, Soares P, Preto A, et al. Type and prevalence of BRAF mutations are closely associated to papillary thyroid carcinoma histotype and patients' age but not with tumour aggressiveness. Virchows Arch 2005;446:589-595.

71 Tallini G, Hsueh A, Liu S, et al. Frequent chromosomal DNA unbalance in thyroid oncocytic (Hurthle cell) neoplasms detected by comparative genomic hybridization. Lab Invest 1999;79:547-555.

72 Evans HL, Vassilopoulou-Sellin R. Follicular and Hurthle cell carcinomas of the thyroid: a comparative study. Am J Surg Pathol 1998;22:1512-1520.

73 Couto JP, Prazeres H, Castro P, et al. How molecular pathology is changing and will change the therapeutics of patients with follicular cell-derived thyroid cancer. J Clin Pathol 2009;62:414-421. 